\title{
What Triggers Catch-Up Saccades During Visual Tracking?
}

\author{
SOPHIE DE BROUWER, ${ }^{1,2}$ DEMET YUKSEL, ${ }^{2}$ GUNNAR BLOHM, ${ }^{1,2}$ MARCUS MISSAL, ${ }^{3}$ AND \\ PHILIPPE LEFÈVRE ${ }^{1,2}$ \\ ${ }^{1}$ Centre for Systems Engineering and Applied Mechanics, Université Catholique de Louvain, B-1348 Louvain-la-Neuve; \\ ${ }^{2}$ Laboratory of Neurophysiology, Université Catholique de Louvain, B-1200 Brussels, Belgium; and ${ }^{3}$ Smith Kettlewell \\ Eye Research Institute, San Francisco, California 94115
}

Received 29 May 2001; accepted in final form 2 November 2001

\begin{abstract}
de Brouwer, Sophie, Demet Yuksel, Gunnar Blohm, Marcus Missal, and Philippe Lefèvre. What triggers catch-up saccades during visual tracking? J Neurophysiol 87: 1646-1650, 2002; 10.1152/jn. 00432.2001. When tracking moving visual stimuli, primates orient their visual axis by combining two kinds of eye movements, smooth pursuit and saccades, that have very different dynamics. Yet, the mechanisms that govern the decision to switch from one type of eye movement to the other are still poorly understood, even though they could bring a significant contribution to the understanding of how the CNS combines different kinds of control strategies to achieve a common motor and sensory goal. In this study, we investigated the oculomotor responses to a large range of different combinations of position error and velocity error during visual tracking of moving stimuli in humans. We found that the oculomotor system uses a prediction of the time at which the eye trajectory will cross the target, defined as the "eye crossing time" $\left(T_{\mathrm{XE}}\right)$. The eye crossing time, which depends on both position error and velocity error, is the criterion used to switch between smooth and saccadic pursuit, i.e., to trigger catch-up saccades. On average, for $T_{\mathrm{XE}}$ between 40 and 180 $\mathrm{ms}$, no saccade is triggered and target tracking remains purely smooth. Conversely, when $T_{\mathrm{XE}}$ becomes smaller than $40 \mathrm{~ms}$ or larger than 180 $\mathrm{ms}$, a saccade is triggered after a short latency (around $125 \mathrm{~ms}$ ).
\end{abstract}

\section{N T R O D U C T I O N}

The retina of primates contains a higher acuity zone or fovea that needs to be precisely oriented to allow a fine perception. Therefore these species developed a repertoire of eye movements to orient the visual axis or gaze toward targets of interest. Saccades are fast eye movements (maximum eye velocity $>500 \mathrm{deg} / \mathrm{s}$ ) that allow primates to shift gaze between stationary targets (Becker 1991). The sensory information the saccadic system uses is the difference between target and gaze positions, i.e., position error. The delays inherent to the processing of visual information prevent from controlling these fast movements under visual feedback. Their control is based on an efference copy of oculomotor commands. Smooth pursuit eye movements allow primates to follow moving objects with the eyes. Smooth pursuit eye movements are much slower than saccades (eye velocity usually $<50 \mathrm{deg} / \mathrm{s}$ ) and are controlled by visual feedback (Pola and Wyatt 1991). The oculomotor system cannot rely solely on position error to orient toward moving targets. Indeed, visual information about target motion is necessary for accurate visual tracking. The sensory

\footnotetext{
Address for reprint requests: P. Lefèvre, CESAME, Université Catholique de Louvain, 4 av. G. Lemaître, B-1348 Louvain-la-Neuve, Belgium (E-mail: lefevre@csam.ucl.ac.be).
}

information used is the relative motion of the target with respect to the fovea, or retinal slip. In natural circumstances, given the delays present in the visual pathways and the saturation of eye acceleration during smooth eye movements (Lisberger and Westbrook 1985; Tychsen and Lisberger 1986), it is necessary to combine smooth eye movements with "catch-up" saccades to catch a moving target. Saccades are particularly frequent during pursuit with a low gain or following unexpected changes in velocity and/or direction of the target (Boman and Hotson 1992). Smooth pursuit eye movements without saccades are typical steady-state responses to predictable target motion (Barnes and Asselman 1991). For pursuit initiation, Rashbass (1961) proposed a paradigm that combines the initial ramp motion of the target with a step in the opposite direction. This paradigm allows varying the size of the initial catch-up saccade or obtaining pursuit initiation without any saccade, depending on the parameters used for the target step and ramp.

The goal of this study is to understand precisely the sensory conditions leading to the occurrence of catch-up saccades during smooth pursuit. Sensory cues available are position error and retinal slip, which are known to play different roles in saccades and smooth pursuit. How the oculomotor system achieves this complex task of decision between two different control strategies, i.e., saccadic and smooth eye movements, is still unknown. This study is a good testing bench for understanding these mechanisms and could give insight concerning the way the CNS combines different control strategies to achieve a common goal.

\section{METHODS}

Subjects were seated $1 \mathrm{~m}$ in front of a tangent screen, which spanned \pm 45 deg of their visual field. Their head was restrained by a chin-rest. The target was a laser spot back-projected onto a screen that moved horizontally. The position of one eye was recorded with the scleral search coil technique (Collewijn et al. 1975; Robinson 1963). Six normal human subjects participated in the experiment, two of them being completely naïve. All procedures were approved by the Université catholique de Louvain ethics committee. All trials started with an initial fixation period of $1 \mathrm{~s}$ at a position $20 \mathrm{deg}$ to the left or to the right of the straight ahead position. The left initial position preceded motion to the right and the right position motion to the left.

\footnotetext{
The costs of publication of this article were defrayed in part by the payment of page charges. The article must therefore be hereby marked "advertisement" in accordance with 18 U.S.C. Section 1734 solely to indicate this fact.
} 
The fixation period was followed by a classical step-ramp target motion lasting $\geq 600 \mathrm{~ms}$ (Rashbass paradigm). We tested three different initial target velocities $\left(T V_{1}=10,20\right.$, or $\left.30 \mathrm{deg} / \mathrm{s}\right)$ in the two horizontal directions. Velocity and direction of the first ramp varied randomly. After a random period varying in a range of $500 \mathrm{~ms}$, a second step in position (PS) and velocity (VS) occurred. Target velocity $\left(T V_{2}=T V_{1}+\mathrm{VS}\right)$ remained constant for another period of minimum 500-ms duration (range: $500-700 \mathrm{~ms}$ ) before a final fixation period $(1,000 \mathrm{~ms})$. We tested a large range of VS (from -50 to +50 $\mathrm{deg} / \mathrm{s})$. For VS larger than $10 \mathrm{deg} / \mathrm{s}$, PS was randomly chosen between -20 and $10 \mathrm{deg}$. For VS smaller than $-10 \mathrm{deg} / \mathrm{s}$, PS was between -10 and $20 \mathrm{deg}$ and for VS between -10 and $10 \mathrm{deg} / \mathrm{s}$, PS was between -10 and $10 \mathrm{deg}$. Subjects were instructed to follow the target. Saccades were detected by an acceleration criterion (750 deg/ $\mathrm{s}^{2}$ ) and were visually inspected. We analyzed only the first saccade occurring after the change in target velocity. Trials with saccades occurring directly after the step (latency $<150 \mathrm{~ms}$ ) were not included in the analysis because their programming could be based on visual information before the step. Trials were first classified in two categories: trials without any saccade in the first $400 \mathrm{~ms}$ following the step ("smooth trials") and trials with the first saccade occurring between 150 and $400 \mathrm{~ms}$ after the step ("saccade trials"). Saccade trials were then subdivided in two categories: trials with the first saccade occurring between 150 and $300 \mathrm{~ms}$ after the step ("early saccade trials") and trials with the first saccade occurring between 300 and $400 \mathrm{~ms}$ after the step ("late saccade trials"). Late saccade trials were of particular interest because their latency was significantly larger than the latency of normal saccades (approximately $200 \mathrm{~ms}$ ), which indicates that the sensory conditions for the triggering of saccades were not present just after the step for these trials.

\section{R E S U L T S}

In our protocol, we used the well-known Rashbass paradigm to initiate pursuit without saccades. After pursuit was initiated and eye velocity reached a steady state, we introduced a second position (PS) and/or velocity step (VS). With this paradigm, velocity and position steps of different amplitudes could be tested during ongoing pursuit (see Fig. 1, $A-H$ ). Examples of smooth pursuit with a target acceleration (VS $>0$ ) are illustrated in the left column of Fig. 1, whereas examples with a target deceleration are represented in the right column of Fig. 1. Both forward target steps (PS $>0$; Fig. $1, A, D, F$, and $H$ ) and reverse target steps (PS $<0$; Fig. $1, B, C, E$, and $G$ ) were tested. When target acceleration was combined with a forward step of the target, a short latency saccade was triggered (Fig. $1 A$, early saccade). The same occurred when the target decelerated and a reverse step in position was applied (Fig. 1B). These early saccade trials are similar to what has been classically reported in the literature (Collewijn and Tamminga 1984) following unexpected changes in target motion. In contrast, in the particular case when the position step was opposite to the velocity step, it was possible to obtain pursuit responses without any saccade (smooth trials) by choosing the appropriate PS, even for very large VS (up to $\pm 50 \mathrm{deg} / \mathrm{s}$ ). This led to smooth eye acceleration (Fig. $1 C$ ) or deceleration (Fig. 1D) in smooth trials. The example in Fig. $1 D$ is of special interest because it illustrates the capability of the smooth pursuit system to change the direction of the eye from right to left with a smooth acceleration. We will refer to these smooth trials as responses to the generalized Rashbass paradigm, in reference to the paradigm proposed by Rashbass (1961) for pursuit initiation. For different combinations of PS and VS, Fig. 1, $E-H$, shows examples of late saccade trials for which smooth eye move-
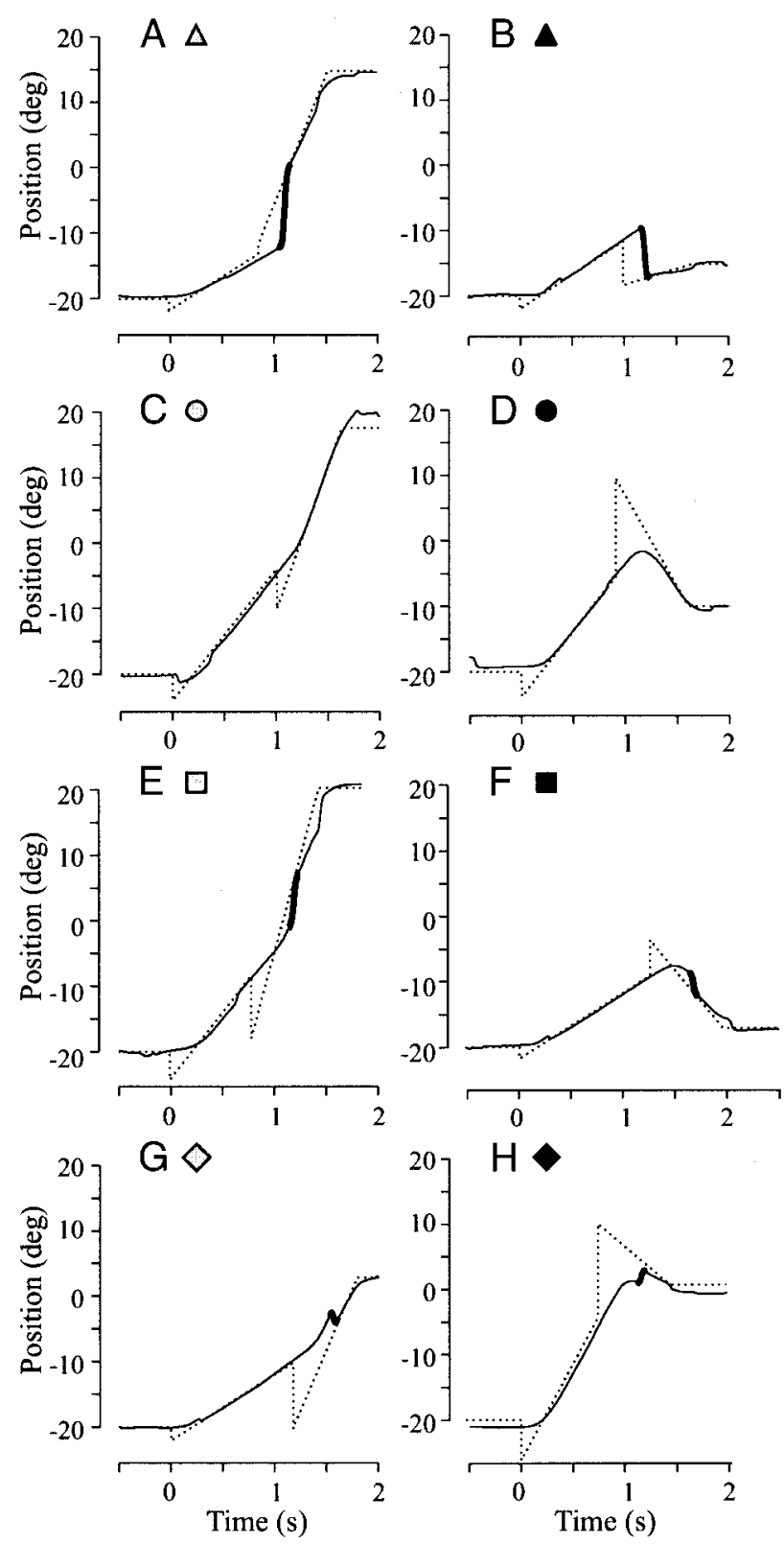

FIG. 1. Examples of responses to unexpected changes in target motion. Eye (target) position is plotted in solid (dotted) line. The first catch-up saccade occurring after the second step is represented by a thick line $(A-B, E-H)$. Examples associated with gray symbols (left) are typical responses to an increase in target velocity between the two ramps, whereas black symbols (right) correspond to responses to a decrease in target velocity. The two first examples $(A$ and $B)$ are early saccade trials for which the position step (PS) and the velocity step (VS) have the same sign. $A$ : response to a positive step combined with an acceleration of the target (PS $>0$, VS $>0$ ), whereas $B$ shows the response to negative PS and VS. Examples in $C$ and $D$ are smooth trials, for which the combination of PS and VS was such that smooth eye movements were sufficient to catch the target. $C$ : smooth eye acceleration (VS $>0$ ); $D$ : smooth deceleration and change in movement direction (VS $<0$ ). The four last panels $(E-H)$ correspond to late saccade trials. In $E$ (respectively, $F$ ), the smooth eye acceleration (respectively, deceleration) is too low and a saccade is necessary to catch the target. In $G$ (respectively, $H$ ), the smooth eye acceleration (respectively, deceleration) is too high and a saccade occurs.

ments did not match target motion and a saccade was triggered to catch the target. This was the case when the smooth response was too weak, i.e., insufficient eye acceleration (Fig. 1E) or deceleration (Fig. $1 F$ ), but also when it was too strong, i.e., too 
high eye acceleration (Fig. 1G) or deceleration (Fig. 1H). In these cases, a late saccade occurred to catch the target by reducing residual position error.

Figure 2 provides, on the basis of sensory signals, an explanation of the mechanism that triggers catch-up saccades. Figure $2 \mathrm{~A}$ illustrates the combinations of retinal slip (RS) and position error (PE) $125 \mathrm{~ms}$ before saccade onset for all saccade trials (gray disks). The duration of $125 \mathrm{~ms}$ was assumed to be the average saccade latency after the decision has been made to trigger a saccade on the basis of RS and PE. This duration is slightly larger than the minimal latency (around $100 \mathrm{~ms}$ ) that is necessary to program a saccade (Becker and Jurgens 1979). Quantitatively similar results are due for latencies in the range of $100-150 \mathrm{~ms}$.

The different symbols correspond to the saccade trials illustrated in Fig. 1, $A, B, E-H$. The saccade zone, which is defined as the domain of retinal slip and position error that contains a larger proportion of saccade trials than smooth trials, includes all but the central sectors of Fig. $2 A$, delimited by the two solid lines. The limits of the saccade zone were determined on the basis of the quantitative analysis illustrated in Fig. 3. Figure $2 B$

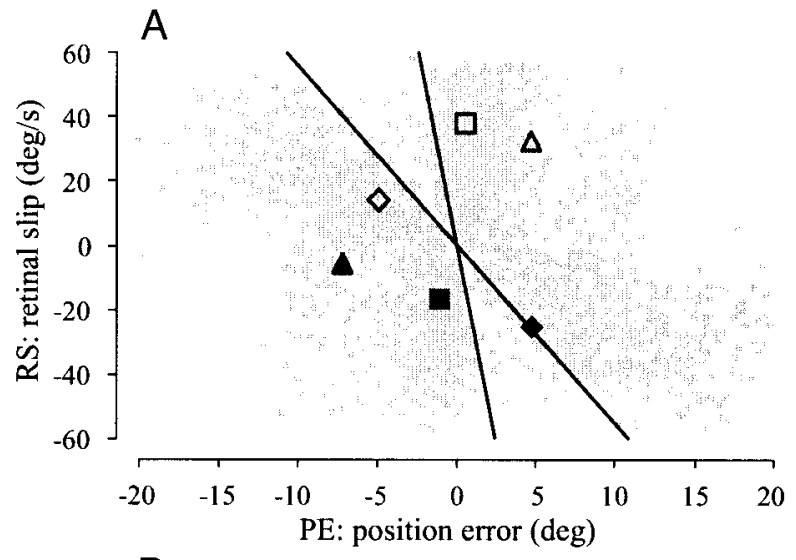

$\mathrm{B}$

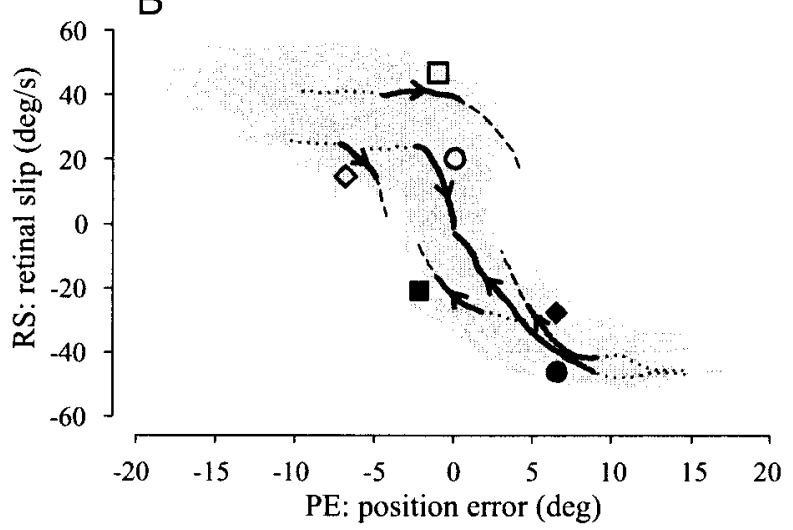

FIG. 2. Role played by position error and retinal slip in saccade trigger. For saccade trials, $A$ plots the combinations of position error (PE) and retinal slip (RS) $125 \mathrm{~ms}$ before saccade onset (gray dots, $n=2733$ ). Data are scattered in the saccade zone, delimited by the two lines with equations: RS $=-\mathrm{PE} / 0.04$ and $\mathrm{RS}=-\mathrm{PE} / 0.18$. The 6 symbols correspond to the saccade trials in Fig. 1 . In $B$, instantaneous RS versus instantaneous $\mathrm{PE}$ is displayed for all smooth trials from the target step to $400 \mathrm{~ms}$ after the step (gray zone). The phase plots of 6 individual examples of Fig. $1, C-H$, are represented next to the associated symbol for clarity. The first $125 \mathrm{~ms}$ after the step are represented in dotted lines, whereas the last $125 \mathrm{~ms}$ before the saccade are in dashed lines (for late saccade trials, Fig. $1, E-H)$ and the rest is in solid lines. Arrows placed along the curves indicate increasing time.

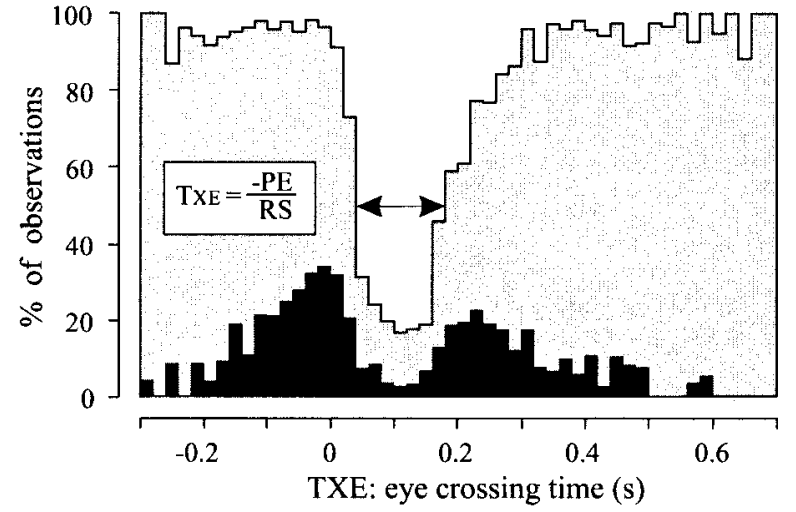

FIG. 3. Quantitative analysis of the limits between smooth and saccadic zones. The relative number of saccade trials (gray histogram, $n=2733$ ) is illustrated as a function of the eye crossing time $T_{\mathrm{XE}}=-\mathrm{PE} / \mathrm{RS}$ (as illustrated in the inset). The black histogram shows the proportion of late saccade trials $(n=542)$. Bins of $20 \mathrm{~ms}$ are represented. The double arrow shows the limits of the smooth zone (40 ms $\left.<T_{\mathrm{XE}}<180 \mathrm{~ms}\right)$.

shows the phase plot of instantaneous RS versus PE for all smooth trials (gray curves). Each segment is associated with a single trial and represents the instantaneous value of RS as a function of PE, starting at the time of the step for a duration of $400 \mathrm{~ms}$. The phase plots of the particular examples of late saccade and smooth trials illustrated in Fig. 1 are superposed (black curves). This panel can be regarded as the complement of panel $A$, as it represents all the combinations of PE and RS that did not trigger a saccade (smooth zone). When the trajectory remains in the middle of the smooth zone, there is a high probability of having a smooth trial (circles corresponding to examples in Fig. 1, $C$ and $D$ ), whereas if the trajectory leaves the gray zone and enters the saccade zone (Fig. 2A), a late saccade occurs (diamonds and squares corresponding to Fig. 1, $E-H$ ). For smooth trials (circles, Fig. $1, C$ and $D$ ), the phase plots end at the origin of the graph, which means that the smooth eye movement catches the target $(\mathrm{PE}=\mathrm{RS}=0)$. For late saccade trials, the transition to dashed lines (Fig. 2B) corresponds to the time at which the decision was made to trigger a saccade. Thus the dashed lines correspond to the last $125 \mathrm{~ms}$ before saccade onset. The interest of the phase plot representation in Fig. $2 B$ is that it shows the eye trajectory as a function of the two parameters playing a role for the saccade trigger ( $\mathrm{PE}$ and $\mathrm{RS}$ ). In the six trajectories of the individual examples in the phase plot, there is no variation of retinal slip in the first $125 \mathrm{~ms}$ after the target step (dotted line), which is consistent with the hypothesis that the information about target step is not yet available to the pursuit system due to visual delays (Becker and Jurgens 1979).

Figure 3 shows the distribution of the relative number of saccade trials as a function of the "eye crossing time," which is the time necessary for the eye to cross the target at constant eye velocity $\left(T_{\mathrm{XE}}=-\mathrm{PE} / \mathrm{RS}\right)$. For saccade trials, $T_{\mathrm{XE}}$ was evaluated on the basis of the retinal slip and position error $125 \mathrm{~ms}$ before saccade onset (Fig. 2A), whereas for smooth trials, each trial was characterized by the average value of $T_{\mathrm{XE}}$ across the smooth trajectory (Fig. 2B, starting at the step and ending 400 $\mathrm{ms}$ after the step). The distribution of the percentage of saccade trials is represented by the gray histogram in Fig. 3, with a minimum below $20 \%$ for $T_{\mathrm{XE}}$ in the range of 80 to $160 \mathrm{~ms}$. The black histogram in Fig. 3 represents the distribution of the 
relative number of late saccade trials. Late saccade trials are concentrated near the borders of the saccade zone, which is delimited by $T_{\mathrm{XE}}=40 \mathrm{~ms}$ and $T_{\mathrm{XE}}=180 \mathrm{~ms}$ (50\% saccade trials, Fig. 2A). For individual subjects, the values of $T_{\mathrm{XE}}$ delimiting the saccade zone varied in the range of 0 to $220 \mathrm{~ms}$.

\section{I S C U S S I O N}

This study brings new insights to the interaction between the saccadic and smooth pursuit systems. Indeed, understanding what the sensory parameters are that determine the triggering of catch-up saccades is fundamental when studying visual tracking of moving targets. We showed that it was possible to control the probability of occurrence of catch-up saccades by combining appropriate values of position and velocity steps. Previous studies were performed with similar experimental conditions (Carl and Gellman 1987; Lisberger et al. 1981; Morris and Lisberger 1987) but they focused on the smooth responses rather than on the mechanism controlling the decision to trigger a catch-up saccade. In our protocol, other combinations of PS and VS that are aimed at triggering early or late saccades would be very useful in studying specifically catch-up saccades during pursuit. Manipulating appropriately PS and VS would provide a wide range of catch-up saccade amplitude for different combinations of RS and PE. This would allow us to get insight on the role played by prediction in catch-up saccades (de Brouwer et al. 2001; Keller and Johnsen 1990; Kim et al. 1997) and could be combined with electrophysiological recordings to better understand the mechanisms of interaction between saccades and smooth pursuit (Keller et al. 1996; Krauzlis et al. 1997).

There are other examples of close interactions between the saccadic and smooth pursuit systems that have been recently described in the literature. It has been shown that there is a common motor or position error signal in the superior colliculus that could be shared by the saccadic and smooth pursuit systems (Basso et al. 2000; Krauzlis et al. 1997, 2000). At the premotor level, Missal et al. (2000) have found neurons in the mesencephalon that are active during both kinds of eye movements, raising the possibility that they play a role in the synergy between saccadic and smooth pursuit systems. Another site of interaction between both systems is the oculomotor cerebellar vermis where lesions affect both saccades and smooth pursuit (Takagi et al. 1998, 2000). Krauzlis and Miles (1998) reported that the vermis might contribute to the elaboration of an error signal that is common to both types of eye movements. Electrical stimulation in that structure evokes saccades or smooth eye movements, depending on the sensory context at the time of stimulation. In our study, the sensory context could be described by the combination of PE and RS. Finally, at the cortical level, there is anatomical evidence for connections between structures containing subregions for saccades and pursuit (Tian and Lynch 1996).

The dynamics and the control mechanisms of smooth and saccadic eye movements are very different. The capacity of the CNS to manage the transitions between these two control modes is a good example of planning in the motor system. A comprehension of this switching mechanism toward a common goal in the oculomotor system might be very useful in understanding the interaction between complex control modes in other motor systems. There are similarities in the control of smooth pursuit and manual tracking of moving targets (Engel et al. 2000) despite the large difference in the inertia of the eye and the arm. After the removal of the saccades from the oculomotor records, it appears that the smooth eye movements and tracking arm movements have similar patterns of responses to changes in target direction. Thus the generalized Rashbass paradigm might also be useful for studying manual tracking.

Our study answered our original question as to "what triggers catch-up saccades?". We found that the main parameter controlling the decision mechanism is the eye crossing time, which depends on two sensory signals: position error and retinal slip. In fact, catch-up saccades are triggered when the value of the eye crossing time enters the saccade zone $\left(T_{\mathrm{XE}}<\right.$ $40 \mathrm{~ms}$ or $T_{\mathrm{XE}}>180 \mathrm{~ms}$ ), which means that it is likely not possible to catch the target solely by means of a purely smooth acceleration. All types of trials (smooth, early, or late saccade trials) obey this same rule. For early saccades, the eye crossing time is already inside the saccade zone soon after the step such that a saccade is triggered rapidly. For late saccades, the eye crossing time first lies within the smooth zone but after some time the pursuit response is such that the trajectory crosses the limit of the saccade zone. In smooth trials, the pursuit response is such that the trajectory remains inside the smooth zone. The eye crossing time parameter that controls the decision to trigger saccades could also be related to the time-to-collision parameter used in steering control (limbs, whole body, cars, or aircraft) (Lee 1998; Regan and Gray 2000). In conclusion, our finding can therefore be considered as a model of how the decision to trigger a saccade could be elaborated during pursuit.

This work was supported by the Fonds National de la Recherche Scientifique; the Belgian program on inter-university poles of attraction initiated by the Belgian state, Prime Minister's office for Science, Technology, and Culture (SSTC); Actions de Recherche Concertées; and an internal research grant (Fonds Spéciaux de Recherche) of the Université Catholique de Louvain.

\section{REFERENCES}

BARNES GR AND ASSELMAN PT. The mechanism of prediction in human smooth pursuit eye movements. J Physiol (Lond) 439: 439-461, 1991.

Basso MA, Krauzlis RJ, AND Wurtz RH. Activation and inactivation of rostral superior colliculus neurons during smooth-pursuit eye movements in monkeys. J Neurophysiol 84: 892-908, 2000.

BECKER W. Saccades. In: Eye Movements: Vision and Visual Dysfunction, edited by Carpenter R. Houndmills, UK: Macmillan Press, 1991, p. 95-137.

BeCKer W AND JuRgens R. An analysis of the saccadic system by means of double step stimuli. Vision Res 19: 967-983, 1979.

Boman DK AND Hotson JR. Predictive smooth pursuit eye movements near abrupt changes in motion direction. Vision Res 32: 675-689, 1992.

CARL JR AND GEllman RS. Human smooth pursuit: stimulus-dependent responses. J Neurophysiol 57: 1446-1463, 1987.

Collewisn H AND TAMminga EP. Human smooth and saccadic eye movements during voluntary pursuit of different target motions on different backgrounds. J Physiol (Lond) 351: 217-250, 1984.

COLlewiJn H, van DER MARK F, AND JANSEN TC. Precise recording of human eye movements. Vision Res 15: 447-450, 1975.

DE Brouwer S, Missal M, AND Lefèvre P. Role of retinal slip in the prediction of target motion during smooth and saccadic pursuit. J Neurophysiol 86: 550-558, 2001.

Engel KC, Anderson JH, And Soechting JF. Similarity in the response of smooth pursuit and manual tracking to a change in the direction of target motion. J Neurophysiol 84: 1149-1156, 2000.

KELLER E AND JOHNSEN SD. Velocity prediction in corrective saccades during smooth-pursuit eye movements in monkey. Exp Brain Res 80: 525-531, 1990 . 
KEller EL, GANDHI NJ, AND WEIR PT. Discharge of superior collicular neurons during saccades made to moving targets. J Neurophysiol 76: 35733577, 1996.

Kim CE, Thaker GK, Ross DE, And Medoff D. Accuracies of saccades to moving targets during pursuit initiation and maintenance. Exp Brain Res 113: 371-377, 1997.

KraUZLIS RJ, BASSO MA, AND WuRTZ RH. Shared motor error for multiple eye movements. Science 276: 1693-1695, 1997.

KRAUZLIS RJ, BASSO MA, AND WURTZ RH. Discharge properties of neurons in the rostral superior colliculus of the monkey during smooth-pursuit eye movements. J Neurophysiol 84: 876-891, 2000.

KRAUZlis RJ AND MiLEs FA. Role of the oculomotor vermis in generating pursuit and saccades: effects of microstimulation. J Neurophysiol 80: 20462062, 1998.

LEE DN. Guiding movements by coupling taus. Ecol Psychol 10: 221-250, 1998.

Lisberger SG, Evinger C, Johanson GW, And Fuchs AF. Relationship between eye acceleration and retinal image velocity during foveal smooth pursuit in man and monkey. J Neurophysiol 46: 229-249, 1981.

LisBerger SG AND WeStBROOK LE. Properties of visual inputs that initiate horizontal smooth pursuit eye movements in monkeys. J Neurosci 5: 16621673, 1985.

Missal M, de Brouwer S, Lefìvre P, and Olivier E. Activity of mesencephalic vertical burst neurons during saccades and smooth pursuit. J Neurophysiol 83: 2080-2092, 2000.
Morris EJ AND LiSBERGER SG. Different responses to small visual errors during initiation and maintenance of smooth-pursuit eye movements in monkeys. J Neurophysiol 58: 1351-1369, 1987.

Pola J AND WyatT HJ. Smooth pursuit: response characteristics, stimuli and mechanisms. In: Eye Movements: Vision and Visual Dysfunction, edited by Carpenter R. Houndmills, UK: Macmillan Press, 1991, p. 138-156.

RASHBASS C. The relationship between saccadic and smooth tracking eye movements. J Physiol (Lond) 159: 326-338, 1961.

REGAN D AND GRAY R. Visually guided collision avoidance and collision achievement. Trends Cogn Sci 4: 99-107, 2000.

RoBINSON DA. A method of mesuring eye movement using a scleral search coil in a magnetic field. IEEE Trans Biomed Eng BME-10: 137-145, 1963.

TAKAGI M, ZEE DS, AND TAMARGo RJ. Effects of lesions of the oculomotor vermis on eye movements in primate: saccades. J Neurophysiol 80: 19111931, 1998.

TAKAGi M, ZEE DS, AND TAMARGo RJ. Effects of lesions of the oculomotor cerebellar vermis on eye movements in primate: smooth pursuit. J Neurophysiol 83: 2047-2062, 2000.

TIAN JR AND LYNCH JC. Corticocortical input to the smooth and saccadic eye movement subregions of the frontal eye field in Cebus monkeys. J Neurophysiol 76: 2754-2771, 1996.

TYCHSEN L AND LISBERGER SG. Visual motion processing for the initiation of smooth-pursuit eye movements in humans. J Neurophysiol 56: 953-968, 1986. 